\title{
Overview of the Mutational Landscape in Primary Myelofibrosis and Advances in Novel Therapeutics
}

\author{
Jaleed Ahmed Gilani ${ }^{1 *}$, Muhammad Areeb Ashfaq ${ }^{2}$, Armaghan-e-Rehman \\ Mansoor $^{3}$, Adnan Abdul Jabbar ${ }^{4}$, Tariq Siddiqui', Maliha Khan ${ }^{5}$
}

\begin{abstract}
Primary Myelofibrosis is a BCR-ABL negative myeloproliferative neoplasm with a variety of hematological presentations, including thrombosis, bleeding diathesis and marrow fibrosis. It is estimated to have an incidence of 1.5 per 100,000 people each year. Although JAK2 or MPL mutations are seen in PMF, several other mutations have recently been documented, including mutations in CALR, epigenetic regulators like TET, ASXL1, and 13q deletions. The identification of these mutations has improved the ability to develop novel treatment options. These include JAK inhibitors like ruxolitinib, heat shock protein-90 inhibitors like ganetespib, histone deacetylase inhibitors including panobinostat, pracinostat, vorinostat and givinostat, hypomethylating agents like decitabine, hedgehog inhibitors like glasdegib, PI3K, AKT and mTOR inhibitors like everolimus as well as telomerase inhibitors like imtelstat. Research on novel therapeutic options is being actively pursued in order to expand treatment options for primary myelofibrosis however currently, there is no curative therapy other than allogenic hematopoietic stem cell transplantation (ASCT) which is possible in select patients.
\end{abstract}

Keywords: Primary Myelofibrosis- Thrombocytosis- Myeloproliferative Disorders

Asian Pac J Cancer Prev, 20 (6), 1691-1699

\section{Introduction}

Primary Myelofibrosis (PMF) is a classic chronic myeloproliferative neoplasm (MPN), belonging to the category of BCR-ABL negative MPNs (Hobbs and Rampal, 2015). It is estimated to have an incidence of 1.5 per 100,000 people each year, with a median age at presentation of 67 years (Mesa et al., 1999).

The clinical presentation of PMF includes hepatosplenomegaly, extramedullary hematopoiesis, expression of portal hypertension and potentially debilitating constitutional symptoms. Classical laboratory features include progressive anemia, leukocytosis, leukoerythroblastosis and thrombocytosis though leukopenia and thrombocytopenia can also occur (Scherber et al., 2011).

Currently, there is no curative therapy other than allogenic hematopoietic stem cell transplantation (ASCT). However, only a select minority of patients are eligible for this procedure (Ballen et al., 2010). Hence, the management of such patients is aimed at controlling disease progression and symptoms in order to improve quality of life for patients.

The pathogenesis of PMF involves a variety of mutations, which include the conventional mutations responsible for the majority of diagnosed cases, as well as a multitude of other mutations implicated in a minority of patients. The 2016 World Health Organization (WHO) classification highlights this clonal nature of the disease by defining the presence of JAK2 (V617F) or MPL (W515) mutations as a major diagnostic criterion (Passamonti and Maffioli). In this review, we discuss these conventional driver mutations, other less frequently encountered mutations associated with PMF, and their role in the disease pathogenesis. We also describe novel therapeutic agents currently available for primary myelofibrosis, as well as future directions for the development of these therapies.

\section{Mutations encountered in PMF \\ JAK mutations}

JAK2 is a non-receptor tyrosine kinase that undergoes phosphorylation after ligand interaction. JAK2 V617F, the first driver mutation to be described, is an activating mutation in exon 14 resulting in valine-to-phenylalanine substitution (Tefferi, 2016). These mutations are seen in $50-60 \%$ of patients with PMF or essential thrombocythemia (ET) (Azzato and Bagg, 2015). The 
V617F mutation prevents physiologic inhibition by occupying the pseudokinase domain of JAK2, directly activating the kinase domain (Shan et al., 2014). Activation of downstream targets of this mutation, however, depends on cytokine receptor expression at various levels, particularly of homodimeric type 1 receptors (Lu et al., 2008; Vainchenker et al., 2016). This gain-of-function mutation leads to constitutive action of downstream STAT3/5 signaling. The resultant continuous JAK-STAT signaling contributes to unchecked myeloproliferation which is the hallmark of this disease (Hobbs and Rampal, 2015; Singh, 2015).

\section{MPL mutations}

Another mutation reported in $11 \%$ of PMF patients involves the MPL oncogene on chromosome 1p34, which encodes the thrombopoietin receptor c-MPL (Tefferi, 2010; Singh, 2015). This type 1 cytokine receptor binds thrombopoietin and activates downstream effectors and signaling pathways including STAT3, STAT5 and PI3K/AKT - which is crucial to the survival and proliferation of megakaryocytes (Sasazawa et al., 2015).

The MPL W515L mutation occurs especially in the JAK2 V617F-negative subset of patients, and causes cytokine-independent activation of the thrombopoietin receptor (Pikman et al., 2006). Subsequent dysregulation of megakaryocyte differentiation and multiplication via overactive JAK-STAT signaling produces a myelofibrosis phenotype (Pikman et al., 2006; Defour et al., 2016).

A similar mutation has also been described where a try-lys substitution at the same codon results in cytokine independent c-MPL receptor activation, (Pardanani et al., 2006).

\section{CALR mutations}

Calreticulin gene (CALR) mutations have been implicated in PMF pathogenesis in JAK2 V617F- and MPL-negative patients (Klampfl et al., 2013). More than 50 frameshift mutations have been reported in exon 9 of CALR, all of which produce a mutant CALR protein with basic charge and loss of binding sites (Klampfl et al., 2013; Nangalia et al., 2013; Tefferi et al., 2014c; Azzato and Bagg, 2015).

The most frequent of these is del52, which is a 'type 1 ' mutation representing $55 \%$ of CALR mutations, and results in loss of all negative charge of the protein generated. On the other hand, the 'type 2' mutation, ins5, is seen in $30 \%$ of CALR-mutated cases and the resultant protein product retains half the negative charges of the normal protein (Klampfl et al., 2013; Vainchenker et al., 2016). Corresponding to these types of mutations, other newly discovered genetic aberrations are classified as 'type 1'- or 'type 2'-like (Vainchenker et al., 2016), with the former being the more common variant (75\% vs $15 \%$ ) (Vainchenker and Kralovics, 2017). A recent meta-analysis found the overall frequency of CALR mutations in PMF patients to be $22 \%$ (Kong et al., 2016).

CALR is located in the endoplasmic reticulum (ER) and serves as a major component of the quality-control machinery by ensuring correct glycoprotein folding. It also contributes to calcium homeostasis, and can bind
$\mathrm{N}$-glycosylated residues of several proteins (Michalak et al., 2009). Preliminary studies suggest that del52 mutations activate STAT5 resulting in cytokine-independent cell growth; mutant CALR has been shown to bind and activate the MPL receptor (Araki et al., 2016; Chachoua et al., 2016). This binding and activation of MPL by mutant CALR can potentially occur at any location between the ER and cell membrane (Araki et al., 2016; Chachoua et al., 2016). Furthermore, since CALR mutants are secreted from the cell, they can also stimulate other cells such as monocytes to produce pro-inflammatory cytokines (Garbati et al., 2016).

A recent study demonstrated that mutant CALR may be considered a MPN-specific tumor antigen, suggesting CALR to be a rational target of immunotherapy. It also showed that the limited $T$ cell response to CALR-mutated MPN involved checkpoint signaling, and that checkpoint inhibitor therapy may be effective in these patients (Cimen Bozkus et al., 2017).

\section{Heterogeneous mutations seen in PMF}

An estimated $10-15 \%$ of patients with PMF do not harbor any of the 3 major mutations described above, and are so called 'triple-negative' (Tefferi et al., 2014b). These patients likely have a range of heterogeneous driver mutations, a large number of which have been correlated with hyperactive JAK/STAT signaling (Rampal et al., 2014). This subset of PMF patients has the poorest prognosis (Tefferi et al., 2014a).

Certain mutations in epigenetic regulator genes have been documented in myelofibrosis, including TET2, ASXL1, EZH2, DNMT3A, and IDH1/IDH2 (Singh). Additionally, spliceosome mutations have a role in the progression of myelofibrosis (Yoshida et al., 2011; Tefferi et al., 2014a).

TET (Ten-Eleven-Translocation) enzymes, consisting of TET1/2/3, are responsible for hydroxylating methylcytosine residues (Rampal and Levine, 2014). TET2 mutations are the most frequently occurring mutations in myelofibrosis not involving the JAK/STAT pathway (Tefferi et al., 2009). In patients harboring both aberrations, TET disruption may precede or follow JAK2 mutations (Rampal and Levine, 2014).

Malignant myeloid diseases frequently have ASXL1 gene mutations (Carbuccia et al., 2009; Gelsi-Boyer et al., 2009). The ASXL1 protein interacts with a group of proteins called polycomb proteins, including the polycomb repressive complex 2 (PRC2) (Abdel-Wahab et al., 2012). They participate in regulating transcription via nuclear hormone receptors (Abdel-Wahab et al., 2012; Saeidi, 2016). ASXL1 mutations result in altered histone methylation, leading to increased expression of HoxA9 and HoxA10, and greater malignant potential (Abdel-Wahab et al., 2012).

Recently, ASX has been shown to deubiquitinate histone $\mathrm{H} 2 \mathrm{~A}$, reversing the normal function mediated by the PRC1 complex (Scheuermann et al.). The regulation of target genes such as the HOX is maintained via the balance between these 2 activities, and is essential to normal genetic expression (Sauvageau and Sauvageau, 2010). Around $19-40 \%$ of patients with PMF have ASX mutations 
Table 1. Frequently Occurring Mutations in Primary Myelofibrosis

\begin{tabular}{|c|c|c|c|}
\hline Mutation & Mutation type & Frequency & Pathophysiology \\
\hline JAK2V617F & Activating mutation & $\begin{array}{l}50-60 \% \text { of patients with } \\
\text { ET or PMF }\end{array}$ & $\begin{array}{l}\mathrm{G} \text { to } \mathrm{T} \text { somatic mutation resulting in valine substituting } \\
\text { phenylalanine (Tefferi, 2016) }\end{array}$ \\
\hline Thrombopoetin receptor MPL & Activating mutation & $11 \%$ of patients with PMF & $\begin{array}{l}\text { Mutations in exon } 10 \text { leading to MPL receptor becoming } \\
\text { active and oncogenic (Pikman et al., 2006) }\end{array}$ \\
\hline Calreticulin gene (CALR) & $\begin{array}{l}\text { Abnormal protein activates } \\
\text { MPL receptors }\end{array}$ & $22 \%$ of patients with PMF & $\begin{array}{l}\text { More than } 50 \text { mutations described in exon 9, with } \\
\text { frameshift resulting in absent KDEL sequence } \\
\text { (Klampfl et al., 2013) }\end{array}$ \\
\hline Triple-negative & $\begin{array}{l}\text { JAK2V617F, } \\
\text { TpoR/MPL and CALR } \\
\text { mutation negative }\end{array}$ & $\begin{array}{l}10-15 \% \text { of patients with } \\
\text { PMF or ET; } \\
\text { Poorest prognosis }\end{array}$ & $\begin{array}{l}\text { Heterogeneous, often associated with increased JAK/ } \\
\text { STAT signaling (Tefferi et al., 2014c) }\end{array}$ \\
\hline ASXL1 & $\begin{array}{l}\text { Loss-of-function mutation of } \\
\text { transcription regulator }\end{array}$ & $\begin{array}{l}19-40 \% \text { of patients with } \\
\text { PMF }\end{array}$ & $\begin{array}{l}\text { Located on chromosome 20q11.1; mutations cause } \\
\text { aberrant histone methylation (Singh, 2015) }\end{array}$ \\
\hline
\end{tabular}

(Singh), with CALR-negative, ASXL1-positive patients being at greatest risk of acquiring this mutation (Tefferi et al., 2014c; Singh, 2015).

Chromosomal aberrations may also be responsible for a subset of cases of MPF. A study at the MD Anderson Cancer Center investigated genomes in PMF patients with isolated del (13q) mutations (Mehrotra et al., 2015). Compared to normal karyotype PMF, del (13q) patients had significantly higher degrees of bone marrow fibrosis and splenomegaly; however there was no difference in median overall survival between the two patient populations.

\section{Therapeutic targets in PMF}

A discussion of how epigenetic mutations in PMF impact prognosis thus demonstrates the rationale for modulating epigenetic regulation. The discovery of JAK V617F and other epigenetic mutations has led to a more focused approach to the management of PMF and development of new therapies. This has the potential to reduce the burden of symptoms and morbidity and mortality associated with this disease. Some of the therapies for PMF are outlined in Figure 1 and detailed further below.

\section{Janus kinase inhibitors: \\ Ruxolitinib}

Ruxolitinib is the prototype Janus kinase inhibitor drug that has demonstrated efficacy in reducing both splenic volume and MF symptoms. Ruxolitinib was approved for PMF following the results of two randomized trials showing clear splenic volume reduction (SVR). In the first trial, $41.9 \%$ of patients on ruxolitinib showed an SVR $\geq$ $35 \%$ compared to $0.7 \%$ in placebo at 24 weeks, while in a second trial, $28.5 \%$ of patients receiving ruxolitinib demonstrated $\geq 35 \%$ SVR at 48 weeks vs $0 \%$ with best available therapy $(\mathrm{p}<0.0001)$ (Harrison et al., 2012; Verstovsek et al., 2012). Importantly, responses were noted both in patients with and without a mutated JAK V617F. Moreover, $67.0 \%$ and $79.9 \%$ of patients initially responsive to ruxolitinib therapy in both trials respectively showed long-lasting splenic responses for 48 weeks (Harrison et al., 2012; Verstovsek et al., 2012). Spleen volume reductions additionally correlated with JAK2p. V617F allele burden (Deininger et al., 2015). The most frequently reported adverse events linked to ruxolitinib were anemia and thrombocytopenia (Harrison et al., 2012; Verstovsek et al., 2012).

Final analysis results of the second trial also showed a $33 \%$ decrease in the risk of death with ruxolitinib compared to best available therapy (hazard ratio $=0.67 ; 95 \% \mathrm{CI}$, $0.44-1.02 ; \mathrm{p}=0.06$ ). However, in a study investigating

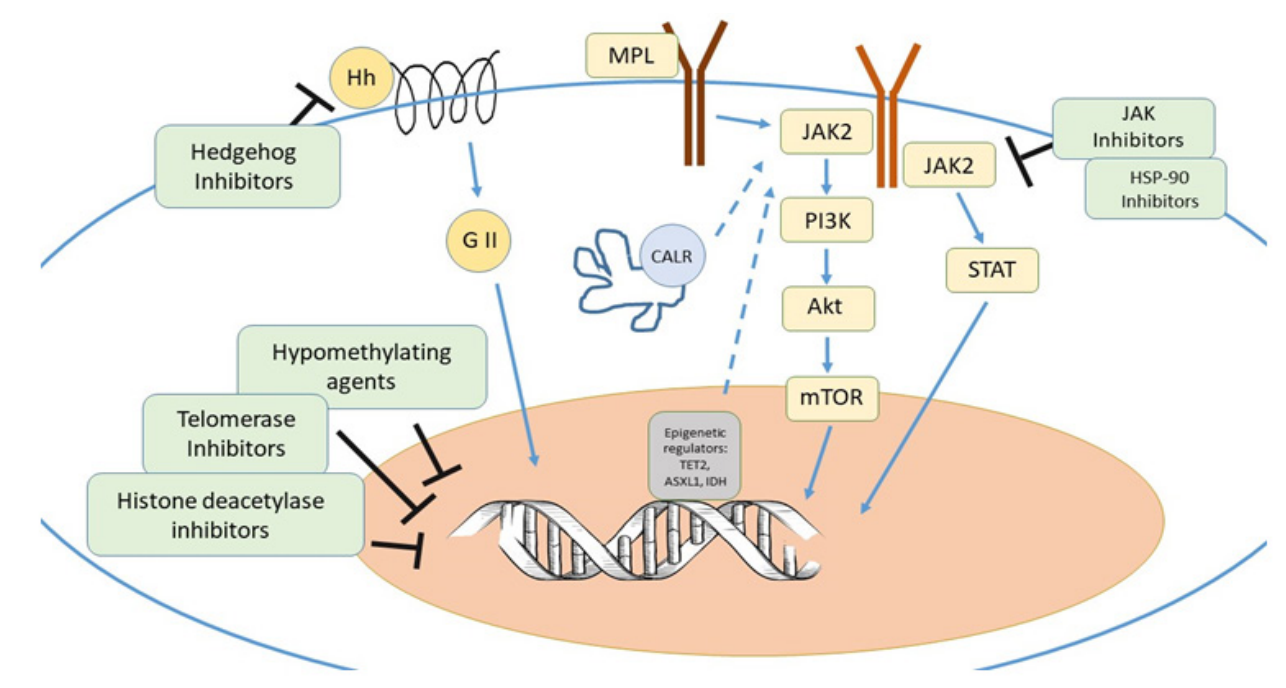

Figure 1. Potential Therapeutic Targets in MF are Illustrated Above 
Table 2. Novel Therapies Investigated in Primary Myelofibrosis

\begin{tabular}{|c|c|c|c|}
\hline Drug & Target & Adverse effects & Study results \\
\hline $\begin{array}{l}\text { JAK2 Inhibitor: } \\
\text { Ruxolitonib }\end{array}$ & $\begin{array}{l}\text { JAK1 } \\
\text { JAK2 }\end{array}$ & $\begin{array}{l}\text { Grade 3-4 anemia, } \\
\text { thrombocytopenia }\end{array}$ & $\begin{array}{l}\text { Primary endpoint of } \geq 35 \% \text { SVR at } 24 \text { and } 48 \text { weeks } \\
\text { seen in } 42 \% \text { and } 28 \% \text { respectively } \\
\text { (Harrison et al., 2016) }\end{array}$ \\
\hline $\begin{array}{l}\text { JAK2 Inhibitor: } \\
\text { Momelotinib (CYT387) }\end{array}$ & $\begin{array}{l}\text { JAK1 } \\
\text { JAK2 }\end{array}$ & Grade 3-4 thrombocytopenia & $\begin{array}{l}\text { Anemia and splenic improvement of } 59 \% \text { and } 48 \\
\text { respectively. } 70 \% \text { of patients dependent on } \\
\text { transfusions achieving independence for } \geq 12 \text { weeks } \\
\text { (Stein et al., 2015). Simplify } 1 / 2 \text { showed } \\
\text { momelotinib falling short in comparison to } \\
\text { ruxolitinib (O'Sullivan and Harrison, 2017) }\end{array}$ \\
\hline $\begin{array}{l}\text { JAK2 Inhibitor: } \\
\text { Pacritinib (SB1518) }\end{array}$ & $\begin{array}{l}\text { JAK2/FLT3 } \\
\text { IRAK1 }\end{array}$ & Gastrointestinal & $\begin{array}{l}\text { PERSIST- } 1 \text { showed significantly improved SVR of } \\
\geq 35 \% \text { at } 24 \text { weeks ( } 19.1 \% \text { of patients on } \\
\text { pacritinib compared to } 4.7 \% \text { with best available } \\
\text { therapy) (Mesa et al.). PERSIST- } 2 \text { showed } \\
\text { significantly better SVR } \geq 35 \% \text { in the pooled } \\
\text { pacritinib arms at } 18 \%, \text { vs } 3 \% \text { with current therapy } \\
(\mathrm{p}=.001) \text { (Mascarenhas et al., } 2016)\end{array}$ \\
\hline $\begin{array}{l}\text { HSP } 90 \text { Inhibitors: } \\
\text { PU-H71, AUY922 }\end{array}$ & JAK2, STAT3, STAT5 & $\begin{array}{l}\text { No clinical data currently } \\
\text { available to report adverse } \\
\text { effects }\end{array}$ & $\begin{array}{l}\text { Preclinical data suggests Hsp90 inhibitors might } \\
\text { improve response compared to JAK inhibition alone } \\
\text { (Fiskus et al., 2011; Proia et al., 2011) }\end{array}$ \\
\hline $\begin{array}{l}\text { Rapamycin, PI3K, and AKT Inhibitors: } \\
\text { Everolimus }\end{array}$ & (mTOR) pathway & $\begin{array}{l}\text { Myelosuppression, } \\
\text { gastrointestinal }\end{array}$ & $\begin{array}{l}\text { Improved activity compared to JAK inhibition alone } \\
\text { (Stein et al., 2015). Ruxolitinib combined with } \\
\text { buparlisib is being evaluated (NCT01730248) }\end{array}$ \\
\hline $\begin{array}{l}\text { Hedgehog Inhibitor: } \\
\text { Sonidegib }\end{array}$ & $\begin{array}{l}\text { JAK-STAT hedgehog } \\
\text { pathways }\end{array}$ & $\begin{array}{l}\text { Grade } 3-4 \text { anemia, } \\
\text { thrombocytopenia }\end{array}$ & $\begin{array}{l}\text { Combination of SMO inhibitor sonidegib with } \\
\text { ruxolitinib showed } \geq 50 \% \text { decrease in } \\
\text { splenomegaly on clinical exam in } 65 \% \text { of MF } \\
\text { patients, with } 9 \text { achieving CR (Gupta et al., 2014) }\end{array}$ \\
\hline $\begin{array}{l}\text { HDAC Inhibitors: } \\
\text { Panobinostat, Givinostat, Pracinostat, } \\
\text { Vorinostat }\end{array}$ & Histone deacetylase & $\begin{array}{l}\text { Grade 3-4 anemia, } \\
\text { thrombocytopenia; } \\
\text { GI side effects with } \\
\text { givinostat; } \\
\text { fatigue and cytopenias in } \\
\text { pracinostat }\end{array}$ & $\begin{array}{l}\text { Panobinostat with ruxolitinib showed }>50 \% \\
\text { reduction in splenomegaly in } 79 \% \text { of patients, with } \\
100 \% \text { reduction in } 53 \% \text { (Kiladjian et al., 2014). } \\
\text { Both drugs in combination are being } \\
\text { currently evaluated (NCT01693601, NCT01433445, } \\
\text { NCT02267278). (Harrison et al., 2015) }\end{array}$ \\
\hline $\begin{array}{l}\text { Telomerase inhibitor: } \\
\text { Imetelstat }\end{array}$ & Telomeres & $\begin{array}{l}\text { Myelosuppression, } \\
\text { grade } 3 \text { anemia, } \\
\text { grade } 4 \text { thrombocytopenia }\end{array}$ & $\begin{array}{l}\text { Imetelstat showed complete or partial remission in } \\
21 \% \text { of primary or secondary to MF patients. } \\
\text { Response was seen only in patients with JAK } 2 \\
\text { mutation (Tefferi et al., 2015). Two large scale } \\
\text { trials (NCT02426086) and (NCT02598661) } \\
\text { currently underway. }\end{array}$ \\
\hline $\begin{array}{l}\text { Hypomethylating agents: } \\
\text { 5- azacytidine, decitabine }\end{array}$ & Epigenetic regulators & $\begin{array}{l}\text { Grade 3-4 neutropenia, } \\
\text { thrombocytopenia, } \\
\text { myelosuppression }\end{array}$ & $\begin{array}{l}\text { In a phase } 2 \text { trial, 5-azacytidine resulted in global } \\
\text { hypomethylation. CR was seen only in } 8 \text { out of } 34 \\
\text { patients and myelosupression was } \\
\text { common (Quintas-Cardama et al., 2008). Future } \\
\text { trials (NCT02076191) are underway. }\end{array}$ \\
\hline
\end{tabular}

SVR, splenic volume reduction; MF, myelofibrosis; CR, complete remission

extended use of the drug noted a discontinuation rate of $92 \%$ at a median of 9.2 months. After discontinuation, severe withdrawal symptoms, labeled "ruxolitinib withdrawal syndrome" were observed. These included accelerated splenomegaly, aggravated cytopenias, and hemodynamic instability resembling septic shock (Tefferi et al., 2011).

\section{Momelotinib (CYT387)}

A combined phase $1 / 2$ study of momelotinib which enrolled 163 patients (64\% of whom had PMF) demonstrated reduction of splenomegaly and induction of durable anemia responses (Pardanani et al., 2011). Unlike other JAK2 inhibitors, momelotinib appeared to improve anemia, a result reproduced in another trial investigating long-term use in 60 patients. Anemia and SVR rates were $59 \%$ and $48 \%$ respectively, and $70 \%$ of patients initially requiring transfusion remained transfusion-independent for at least 12 weeks (Pardanani et al., 2013). Grade 3-4 thrombocytopenia was documented in $32 \%$ of patients.

A recent phase 3 trial comparing momelotinib to ruxolitinib showed achievement of a primary endpoint of $\geq 35 \%$ SVR in $26.5 \%$ patients in the momelotinib arm versus $29 \%$ in the ruxolitinib arm at week 24 , but was unable to demonstrate superiority of momelotinib in splenic size reduction ( $\mathrm{p}=0.111$ ) (O’Sullivan and Harrison, 2017). Due to these overall findings, the development of momelotinib has been aborted (O'Sullivan and Harrison, 2017).

\section{Pacritinib (SB1518)}

Pacritinib exerts its action by inducing JAK2/FLT3 inhibition (Hart et al., 2011), in addition to blocking IRAK1, an IL-1 receptor kinase (Singer et al., 2014). IRAK 1 is overexpressed in both myelodysplastic syndromes and Fanconi anemia, which display markedly deregulated hematopoiesis (Hofmann et al., 2002; Pellagatti et al., 2010). Phase I studies showed minimal myelosuppression, and in results from a phase 2 study with 35 patients, gastrointestinal side effects were most common, notably diarrhea (Komrokji et al., 2015; Jain and Mesa, 2016). Up till week 24, 31\% of patients demonstrated $\mathrm{a} \geq 35 \% \mathrm{SVR}$ on MRI, and $42 \%$ experienced 
reduction in splenic size by $>50 \%$ as gauged by physical examination. Median symptom improvement was $\geq 50 \%$ for all symptoms except fatigue (Komrokji et al., 2015).

Regarding hematological toxicity, pacritinib appears relatively safe as compared to ruxolitinib: only $0.5 \%$ of patients receiving pacritinib experienced grade 3-4 anemia, and $4.2 \%$ experienced grade 3-4 thrombocytopenia (Beauverd et al., 2015). No statistically significant decrease in either hemoglobin levels or platelet count was evident during the treatment course when compared to baseline (Beauverd et al., 2015).

In the phase 3 study PERSIST-1, 327 patients with myelofibrosis were randomized to pacritinib or best available therapy. The primary outcome measure of an SVR of $\geq 35 \%$ was achieved by $19.1 \%$ patients in the pacritinib group versus $4.7 \%$ in the control group $(p=0.0003)$ (Mesa et al., 2017). At week 60, 24\% of evaluable patients receiving pacritinib maintained an SVR of $\geq 35 \%$. The most frequent adverse effects included grade 3 GI symptoms such as diarrhea $(5 \%)$, nausea $(<1 \%)$ and vomiting $(<1 \%)$ (Mesa et al., 2017).

Subsequently, PERSIST-2 was designed to assess two different treatment regimens of pacritinib against standard therapy (Mascarenhas et al., 2016). In the final analysis, significant splenic response ( $\geq 35 \%$ SVR) was seen in the pooled pacritinib arms compared to standard therapy (18\% in pooled pacritinib arms versus $3 \%$ with standard therapy, $\mathrm{p}=.001$ ); however, improved symptom control as determined by significant reduction in total symptom score (by $\geq 50 \%$ ) was not observed with pacritinib therapy (Mascarenhas et al., 2016).

Significant cardiovascular and hemorrhagic events occurred in both PERSIST trials. In particular, increased mortality from cardiac failure, cardiac arrest and intracerebral hemorrhage was seen in the pacritinib arm of PERSIST-1. This led to the FDA halting the drug in February 2016 (Bose et al., 2017). However the ban was lifted in January 2017 following submission of final clinical study reports, and pacritinib is currently under study in another clinical trial (NCT03165734) (Bose et al., 2017).

\section{The challenges of JAK inhibition}

Although JAK inhibitors constitute the basis of treatment for myelofibrosis, there are certain issues associated with their use. For one, myelosuppression, although manageable, is a common toxicity. Secondly, JAK inhibitors are unable to eradicate the mutant JAK. Furthermore, they do not selectively target mutant JAK2 alone, potentially leading to adverse effects including cytopenias that result from disruption of normal JAK signaling (Stein et al., 2015). Impaired dendritic cell function resulting from JAK $1 / \mathrm{JAK} 2$ inhibition can increase susceptibility to infection, while blockade of FLT3 can cause diarrhea (Heine et al., 2013).

In the long-term, JAK/STAT activation can persist despite the use of JAK inhibitors via the formation of heterogenous dimers with other JAK proteins (Koppikar et al., 2012). Finally, the pathogenesis of PMF involves molecular abnormalities at multiple levels which may not all be targeted by a single agent (Stein et al., 2015). This was demonstrated in an in vivo study which showed that resistance to JAK inhibitors can arise via the activation of PDGF-mediated MAPK signaling pathways independent of JAK blockade (Meyer et al., 2017). Further data also suggests that modulating this pathway through the use of MEK inhibitors decreases the activity of MAPK and its downstream pathways (Meyer et al., 2017). This allows for another potential treatment modality for use in PMF.

Other agents which can be used for treatment of MF either alone or in combination with JAK inhibitors are discussed below.

\section{Eliminating resistance to JAK inhibition - HSP-90 inhibitors}

Heat shock proteins (Hsp) represent a group of chaperone molecules tasked with facilitating correct protein folding. In particular, Hsp-90 mediates folding of approximately 200 proteins, many of which are involved in normal cellular signaling (Jhaveri and Modi, 2012). PU-H71 is an Hsp-90 inhibitor that regulates JAK2 expression and thus reduces activation of downstream pathways including STAT3/5, resulting in cell death in both MPL and JAK-mutant clones (Santos and Verstovsek, 2013). In a separate study, another HSP-90 inhibitor, AUY922 decreased mutant JAK expression and increased apoptosis of CD34+ cells (Fiskus et al., 2011; Stein et al., 2015). Ganetespib, another HSP-90 inhibitor, decreased in vitro and in vivo STAT activity (Proia et al., 2011). Hsp-90 inhibition thus may be a potential strategy for overcoming ruxolitinib resistance mechanisms, although at present clinical data is limited.

\section{Addressing epigenetic regulation \\ Histone deacetylase inhibitors}

Panobinostat, pracinostat, vorinostat and givinostat are four drugs in this class that have been investigated in patients with primary myelofibrosis, with the most encouraging preliminary results coming from panobinostat (Mascarenhas et al., 2013; Stein et al., 2015). A phase 1 study showed clinical response in 5 out of $18 \mathrm{PMF}$ patients, of whom 3 had a $100 \%$ reduction in splenomegaly, and 2 had improvement in anemia (Mascarenhas et al., 2017). Panobinostat along with ruxolitinib was also studied in $61 \mathrm{MF}$ patients. An SVR $>50 \%$ was achieved in $79 \%$ of patients, with $53 \%$ having a non-palpable spleen on physical exam (Kiladjian et al., 2014). Adverse events were similar to ruxolitinib monotherapy, i.e. anemia and thrombocytopenia.

In preclinical studies, ruxolitinib and panobinostat showed synergistic activity, with an improvement in fibrosis and reduced bone marrow cellularity (Baffert et al., 2011; Stein et al., 2015). As a result, this combination is now being evaluated in 3 clinical trials (NCT01693601, NCT01433445, and NCT02267278). Preliminary results from one study with 23 patients (NCT01433445) reported a $\geq 35 \%$ SVR in $57 \%$ and $39 \%$ of patients at 24 and 48 weeks respectively, as well as improvement in bone marrow fibrosis in 4 patients. $A \geq 20 \%$ decrease in the JAK2 V617F allele burden was seen in 5 patients at 48 weeks (Harrison et al., 2015). These results show a better outcome than that expected with ruxolitinib alone, and detailed results are awaited. 
In individual phase 2 studies, other histone deacetylase inhibitors tended to have a moderate to low response, and demonstrated adverse effects which restricted their use. Givinostat induced gastrointestinal side effects in $62 \%$ of patients; while several patients on pracinostat experienced fatigue and cytopenias (Rambaldi et al., 2010).

\section{Hypomethylating agents}

ASX1, an epigenetic regulator of MF, is susceptible to hypomethylation. 5-azacytidine and decitabine are two such hypomethylating agents that have been studied in patients with MF. A phase 2 trial with 34 patients given 5 -azacytidine showed hypomethylation in all patients, but clinical improvement was recorded in only 8 patients, and myelosupression was commonly observed (QuintasCardama et al., 2008). Similarly, in 21 patients with myelofibrosis treated with decitabine, a response was seen in 7 of 19 evaluable patients; reduction in spleen size was not reported (Odenike et al., 2008). Grade 3/4 neutropenia and thrombocytopenia was seen in $95 \%$ and $52 \%$ of patients in this cohort (Odenike et al., 2008). Decitabine in combination with ruxolitinib is under investigation at present (NCT02076191).

\section{Drugs targeting other signaling pathways}

Hedgehog inhibitors

Hedgehog proteins are lipid-modified signaling proteins with a final downstream anti-apoptotic effect (Sochacki et al., 2016), and have been shown to interact in MF. Preclinical models showed that granulocytes derived from MPN patients had increased hedgehog target gene expression compared to controls. Murine models treated with ruxolitinib in combination with a smoothened (SMO) inhibitor had lower mutant-allele burden than those treated with either drug alone. Bone marrow fibrosis was also reduced (Keller et al., 2013). In a Phase 1 trial with 23 patients, combination therapy with sonidegib (LDE226), an SMO inhibitor, and ruxolitinib was investigated (Gupta et al., 2014). $65 \%$ of MF patients had a $\geq 50 \%$ reduction in splenomegaly on physical exam, with an impalpable spleen in 9 patients. Anemia and thrombocytopenia were documented in a minority of patients (Gupta et al., 2014).

Glasdegib, another SMO inhibitor is being investigated currently in a Phase $1 \mathrm{~b} / 2$ trial of 21 patients with MF and preliminary results suggest modest clinical activity of this drug. 5 patients showed a spleen volume reduction whilst one patient had improvement in anemia. It should be noted that $52 \%$ of patients $(n=11)$ were refractory to treatment (and had prior JAK inhibitor treatment). Dysgeusia and muscle spasms were frequent side effects (Gerds et al., 2017).

\section{Inhibiting PI3K, AKT and mTOR pathways}

The PI3K/AKT/mTOR pathway functions downstream of JAK/STAT, and thus can serve as a potential target for therapeutic inhibition in MF. This pathway is active in myeloproliferative neoplasms, and acts by dysregulating cell death (Guglielmelli et al., 2011). Everolimus, an mTOR inhibitor, was investigated in a study of 30 patients with myelofibrosis. Clinical improvement was seen in six patients (reduction in spleen volume in 5 patients, raised hemoglobin in 1 patient), while one patient even had a partial remission (Guglielmelli et al., 2011).

Drugs from this class have also been shown to act synergistically when combined with JAK inhibitors (Vannucchi et al., 2011; Bogani et al., 2013). Ruxolitinib in combination with buparlisib (BKM120) has been investigated in a phase 1b trial (NCT01730248), and the results are expected to be made available soon. The AKT inhibitor MK-2206 demonstrated decreased hepatosplenomegaly and megakaryocyte burden in animal models, and decreased megakaryocyte colony formation in patient samples (Khan et al., 2013). When used in conjunction with ruxolitinib, synergistic inhibition of cell growth in JAK mutants was seen, warranting clinical investigation.

\section{Telomerase inhibition: An additional novel strategy}

Repetitive telomere sequences found at the end of chromosomes function to protect coding DNA from genetic damage (Cerquozzi et al., 2016). As cells age, the telomere sequences at the end of their chromosomes become shorter, eventually leading to programmed cell death. There is dramatic upregulation of the telomerase enzyme in malignant cells, conferring unlimited replication potential. This makes the telomerase enzyme a relatively specific target for therapy (Sochacki et al., 2016). The telomerase inhibitor imetelstat was administered to 33 primary/secondary MF patients in a pilot study (Tefferi et al., 2015). 21\% of these patients demonstrated a response, and 4 patients achieved complete remission with bone marrow fibrosis reversal, with 3 of these additionally demonstrating a molecular response. Interestingly, responses were seen only in patients with JAK2 mutation, whereas ASXL1-mutant patients were unaffected. Myelosuppression was the most significant adverse event reported along with grade 3 anemia and grade 4 thrombocytopenia (Tefferi et al., 2015).

Overall, there is significant potential in telomerase inhibitor therapy. Two large scale trials, IMbark (NCT02426086) and IMerge (NCT02598661), studying imtelsat are ongoing at present.

\section{Future perspective}

Despite the multitude of clinical trials investigating various therapies for PMF currently underway, there remains a dearth of information about disease pathogenesis. Further work is needed to elucidate mechanisms of disease initiation, as JAK2 V617F is a mutation that is frequently acquired but does not always lead to disease. Likewise it also remains to be understood why JAK2 V617F and mutant CALR pathways cause distinct (albeit closely related) diseases, even though both activate the same MPL/JAK2 pathway.

Additional gene mutations besides JAK2 V617F are thought to be involved in the pathophysiology of myeloproliferative neoplasms, and additional studies are needed to confirm the pathogenetic role of other phenotypic modifiers such as epigenetic regulators.

As recommended by the 2016 WHO classification of myeloproliferative neoplasms, further research is required 
to fully characterize the influence of molecular findings on disease prognosis. It is particularly relevant in the context of pre-myelofibrosis to determine the impact of CALR/ ASXL1 status on prognosis (Passamonti and Maffioli, 2016).

Given the multifactorial nature of the disease, with many interlinked genetic and environmental elements implicated in its pathophysiology, it remains to be seen which multi-drug regimen will serve as the best treatment of choice for primary myelofibrosis. Hypomethylating agents are a group of epigenetic regulators that may be a promising class of drugs for primary myelofibrosis, especially in combination with ruxolitinib. NS-018 is a JAK inhibitor currently in development, and has shown encouraging results that merit its further investigation. Interferon has also been proposed as a therapeutic option for PMF, but data from this patient population is limited.

Lastly, as discussed in this review, PI3K/mTOR/AKT inhibitors and telomerase inhibitors have also shown much potential, and further studies are warranted to explore this treatment option for primary myelofibrosis.

In conclusion, our understanding of PMF pathogenesis has improved vastly in the past decade, due in major part to the discovery of some key driver mutations that underlie this disease. Recent genomic studies have shown that PMF can progress to a more advanced form of myeloproliferative neoplasia (MPN): a heterogenous disorder that exhibits phenotypic and genotypic features of both myeloproliferative and myelodysplastic syndrome (MDS), called MPN/MDS. Due to this, monotherapy appears to be a suboptimal strategy, thus warranting the development of novel combination approaches for patients with primary myelofibrosis. Several of the new therapeutic options discussed here have shown some promise in early trials, and are expected to be investigated more thoroughly for their potential to reduce the morbidity and mortality associated with this disease.

\section{Executive summary}

- Primary myelofibrosis is a myeloproliferative neoplasm that has a diverse range of underlying genetic causes.

- Genetic mutations in JAK, MPL and CALR genes are common drivers of this disease.

- Mutations in epigenetic regulators such as ASXL1, and spliceosome components, as well as TET enzymes have also been shown to cause disease.

- Some patients, described as "triple negative", do not express any of the 3 major mutations; this subset has the poorest prognosis.

- Allogenic stem cell transplant (ASCT) remains the only definitive cure, but this procedure can only be performed in a selected patients. The associated high morbidity and mortality further points out the urgent need to develop alternative therapeutic modalities.

- JAK2 inhibitors, in particular ruxolitinib, are used to treat PMF and produce significant reduction in splenomegaly, but are associated with significant anemia and thrombocytopenia.

- Significant inherent challenges associated with JAK inhibitor monotherapy warrant the development of new drug modalities and multi-drug regimens.

- Other therapeutic modalities that can potentially be used in conjunction with JAK2 inhibitors include epigenetic modulators, hedgehog inhibitors, inhibitors of $\mathrm{PI} 3 \mathrm{~K} / \mathrm{AKT} / \mathrm{mTOR}$ pathways, and telomerase inhibitors.

- Epigenetic regulator mutations, the target of histone deacetylase inhibitors, have shown significant reduction in splenomegaly when used in combination with JAK inhibitors. Hypomethylating agents however, have limited clinical response.

- The SMO inhibitor sonidegib when used in conjunction with ruxolitinib showed a significant reduction in splenomegaly in approximately two-thirds of patients with PMF in a phase I study.

- $\mathrm{PI} 3 \mathrm{~K} / \mathrm{mTOR}$ inhibitors and JAK $1 / 2$ inhibitors in combination have caused synergistic inhibition of myeloproliferative neoplastic cells.

- Lastly, telomerase inhibitors such as imtelstat have shown encouraging results in pilot studies, though their efficacy is limited to patients with JAK2 mutations alone.

\section{Acknowledgements}

The authors have no conflict of interests to declare. This manuscript did not receive any external funding. No ethical issues were encountered at any stage during the drafting of the manuscript.

\section{References}

Abdel-Wahab O, Adli M, LaFave LM, et al (2012). ASXL1 mutations promote myeloid transformation through loss of PRC2-mediated gene repression. Cancer Cell, 22, 180-93.

Araki M, Yang Y, Masubuchi N, et al (2016). Activation of the thrombopoietin receptor by mutant calreticulin in CALR-mutant myeloproliferative neoplasms. Blood, 127, 1307-16.

Azzato EM, Bagg A (2015). Molecular genetic evaluation of myeloproliferative neoplasms. Int J Lab Hematol, 37, 61-71.

Baffert F, Evrot E, Ebel N, et al (2011). Improved efficacy upon combined JAK1/2 and pan-deacetylase inhibition using Ruxolitinib (INC424) and Panobinostat (LBH589) in preclinical mouse models of $<\mathrm{em}>\mathrm{JAK} 2</ \mathrm{em}>\mathrm{V} 617 \mathrm{~F}$ driven disease. Blood, 118, 798.

Ballen KK, Shrestha S, Sobocinski KA, et al (2010). Outcome of transplantation for myelofibrosis. Biol Blood Marrow Transplant, 16, 358-67.

Beauverd Y, McLornan DP, Harrison CN (2015). Pacritinib: a new agent for the management of myelofibrosis?. Expert Opin Pharmacother, 16, 2381-90.

Bogani C, Bartalucci N, Martinelli S, et al (2013). mTOR inhibitors alone and in combination with JAK2 inhibitors effectively inhibit cells of myeloproliferative neoplasms. PLoS One, 8, e54826.

Bose P, Abou Zahr A, Verstovsek S (2017). Investigational Janus kinase inhibitors in development for myelofibrosis. Expert Opin Investig Drugs, 26, 723-34.

Carbuccia N, Murati A, Trouplin V, et al (2009). Mutations of ASXL1 gene in myeloproliferative neoplasms. Leukemia, 23, 2183-6.

Cerquozzi S, Farhadfar N, Tefferi A (2016). Treatment of myelofibrosis: A moving target. Cancer $J, 22,51-61$.

Chachoua I, Pecquet C, El-Khoury M, et al (2016). 
Thrombopoietin receptor activation by myeloproliferative neoplasm associated calreticulin mutants. Blood, 127, 1325-35.

Cimen Bozkus C, Finnigan JP, Mascarenhas J, et al (2017). Immune checkpoint blockade enhances mutated calreticulininduced T cell Immunity in myeloproliferative neoplasms. Blood, 130, 384-.

Defour JP, Chachoua I, Pecquet C, et al (2016). Oncogenic activation of MPL/thrombopoietin receptor by 17 mutations at W515: implications for myeloproliferative neoplasms. Leukemia, 30, 1214-6.

Deininger M, Radich J, Burn TC, et al (2015). The effect of long-term ruxolitinib treatment on JAK2p.V617F allele burden in patients with myelofibrosis. Blood, 126, 1551-4.

Fiskus W, Verstovsek S, Manshouri T, et al (2011). Heat shock protein 90 inhibitor is synergistic with JAK2 inhibitor and overcomes resistance to JAK2-TKI in human myeloproliferative neoplasm cells. Clin Cancer Res, 17, 7347-58.

Garbati MR, Welgan CA, Landefeld SH, et al (2016). Mutant calreticulin-expressing cells induce monocyte hyperreactivity through a paracrine mechanism. Am J Hematol, 91, 211-9.

Gelsi-Boyer V, Trouplin V, Adelaide J, et al (2009). Mutations of polycomb-associated gene ASXL1 in myelodysplastic syndromes and chronic myelomonocytic leukaemia. $\mathrm{Br} J$ Haematol, 145, 788-800.

Gerds AT, Tauchi T, Ritchie EK, et al (2017). Phase I/II trial of Glasdegib in heavily pre-treated patients with primary or secondary myelofibrosis. Blood, 130, 258-.

Guglielmelli P, Barosi G, Rambaldi A, et al (2011). Safety and efficacy of everolimus, a mTOR inhibitor, as single agent in a phase $1 / 2$ study in patients with myelofibrosis. Blood, 118, 2069-76.

Gupta V, Koschmieder S, Harrison CN, et al (2014). Phase 1b dose-escalation study of sonidegib (LDE225) in combination with Ruxolitinib (INC424) in patients with myelofibrosis. Blood, 124, 712.

Harrison C, Kiladjian JJ, Al-Ali HK, et al (2012). JAK inhibition with ruxolitinib versus best available therapy for myelofibrosis. N Engl J Med, 366, 787-98.

Harrison CN, Kiladjian J-J, Heidel FH, et al (2015). Efficacy, safety, and confirmation of the recommended phase 2 starting dose of the combination of Ruxolitinib (RUX) and Panobinostat (PAN) in patients (Pts) with myelofibrosis (MF). Blood, 126, 4060-.

Harrison CN, Vannucchi AM, Kiladjian JJ, et al (2016). Long-term findings from COMFORT-II, a phase 3 study of ruxolitinib vs best available therapy for myelofibrosis. Leukemia, 30, 1701-7.

Hart S, Goh KC, Novotny-Diermayr V, et al (2011). SB1518, a novel macrocyclic pyrimidine-based JAK2 inhibitor for the treatment of myeloid and lymphoid malignancies. Leukemia, 25, 1751.

Heine A, Held SA, Daecke SN, et al (2013). The JAK-inhibitor ruxolitinib impairs dendritic cell function in vitro and in vivo. Blood, 122, 1192-202.

Hobbs GS, Rampal RK (2015). Clinical and molecular genetic characterization of myelofibrosis. Curr Opin Hematol, 22, 177-83.

Hofmann WK, de Vos S, Komor M, et al (2002). Characterization of gene expression of CD34+ cells from normal and myelodysplastic bone marrow. Blood, 100, 3553-60.

Jain T, Mesa R (2016). The development, safety and efficacy of pacritinib for the treatment of myelofibrosis. Expert Rev Anticancer Ther, 16, 1101-8.

Jhaveri K, Modi S (2012). HSP90 inhibitors for cancer therapy and overcoming drug resistance. Adv Pharmacol, 65, 471517.

Keller MD, Rampal RK, Shank K, et al (2013). Improved Efficacy OfCombination Of JAK2 and Hedgehog Inhibitors In Myelofibrosis. Blood, 122, 666-.

Khan I, Huang Z, Wen Q, et al (2013). AKT is a therapeutic target in myeloproliferative neoplasms. Leukemia, 27, 1882.

Kiladjian J-J, Heidel FH, Vannucchi AM, et al (2014). Efficacy, safety, and confirmation of the recommended phase 2 dose of Ruxolitinib plus panobinostat in patients with intermediate or high-risk myelofibrosis. Blood, 124, 711-.

Klampfl T, Gisslinger H, Harutyunyan AS, et al (2013). Somatic mutations of calreticulin in myeloproliferative neoplasms. N Engl J Med, 369, 2379-90.

Komrokji RS, Seymour JF, Roberts AW, et al (2015). Results of a phase 2 study of pacritinib (SB1518), a JAK2/JAK2(V617F) inhibitor, in patients with myelofibrosis. Blood, 125, 264955.

Kong H, Liu Y, Luo S, et al (2016). Frequency of calreticulin (CALR) mutation and its clinical prognostic significance in essential thrombocythemia and primary myelofibrosis: A meta-analysis. Intern Med, 55, 1977-84.

Koppikar P, Bhagwat N, Kilpivaara O, et al (2012). Heterodimeric JAK-STAT activation as a mechanism of persistence to JAK2 inhibitor therapy. Nature, 489, 155-9.

Lu X, Huang LJ, Lodish HF (2008). Dimerization by a cytokine receptor is necessary for constitutive activation of JAK2V617F. J Biol Chem, 283, 5258-66.

Mascarenhas J, Hoffman R, Talpaz M, et al (2016). Results of the persist- 2 phase 3 study of pacritinib (PAC) versus best available therapy (BAT), including Ruxolitinib (RUX), in patients (pts) with myelofibrosis (MF) and platelet counts \&lt;100,000/ $\mu 1$. Blood, 128, LBA-5-LBA-.

Mascarenhas J, Lu M, Li T, et al (2013). A phase I study of panobinostat (LBH589) in patients with primary myelofibrosis (PMF) and post-polycythaemia vera/essential thrombocythaemia myelofibrosis (post-PV/ET MF). Br J Haematol, 161, 68-75.

Mehrotra M, Patel KP, Chen T, et al (2015). Genomic and clinicopathologic features of primary myelofibrosis with isolated 13q deletion. Clin Lymphoma Myeloma Leuk, 15, 496-505 e1-2.

Mesa RA, Silverstein MN, Jacobsen SJ, et al (1999). Population-based incidence and survival figures in essential thrombocythemia and agnogenic myeloid metaplasia: an Olmsted County Study, 1976-1995. Am J Hematol, 61, 10-5.

Mesa RA, Vannucchi AM, Mead A, et al (year???). Pacritinib versus best available therapy for the treatment of myelofibrosis irrespective of baseline cytopenias (PERSIST-1): an international, randomised, phase 3 trial. Lancet Haematol, 4, 225-36.

Meyer SC, Ghosh N, Stivala S, et al (2017). Targeting cell non-autonomous MAPK activation as a novel therapeutic strategy in myeloproliferative neoplasms. Blood, 130, 381.

Michalak M, Groenendyk J, Szabo E, et al (2009). Calreticulin, a multi-process calcium-buffering chaperone of the endoplasmic reticulum. Biochem J, 417, 651-66.

Nangalia J, Massie CE, Baxter EJ, et al (2013). Somatic CALR mutations in myeloproliferative neoplasms with nonmutated JAK2. N Engl J Med, 369, 2391-405.

O'Sullivan JM, Harrison CN (2017). JAK-STAT signaling in the therapeutic landscape of myeloproliferative neoplasms. Mol Cell Endocrinol, 451, 71-9.

Odenike OM, Godwin JE, Van Besien K, et al (2008). Phase II trial of low dose, subcutaneous decitabine in myelofibrosis. Blood, 112, 2809.

Pardanani A, Gotlib J, Gupta V, et al (2011). An expanded 
multicenter phase I/II study of CYT387, a JAK- 1/2 inhibitor for the treatment of myelofibrosis. Blood, 118, 3849 .

Pardanani A, Laborde RR, Lasho TL, et al (2013). Safety and efficacy of CYT387, a JAK1 and JAK2 inhibitor, in myelofibrosis. Leukemia, 27, 1322-7.

Pardanani AD, Levine RL, Lasho T, et al (2006). MPL515 mutations in myeloproliferative and other myeloid disorders: a study of 1182 patients. Blood, 108, 3472-6.

Passamonti F, Maffioli M (2016). Update from the latest WHO classification of MPNs: a user's manual. Hematol Am Soc Hematol Educ Program, 2016, 534-42.

Pellagatti A, Cazzola M, Giagounidis A, et al (2010). Deregulated gene expression pathways in myelodysplastic syndrome hematopoietic stem cells. Leukemia, 24, 756-64.

Pikman Y, Lee BH, Mercher T, et al (2006). MPLW515L is a novel somatic activating mutation in myelofibrosis with myeloid metaplasia. PLoS Med, 3, e270.

Proia DA, Foley KP, Korbut T, et al (2011). Multifaceted intervention by the Hsp90 inhibitor ganetespib (STA-9090) in cancer cells with activated JAK/STAT signaling. PLoS One, 6, e18552.

Quintas-Cardama A, Tong W, Kantarjian H, et al (2008). A phase II study of 5-azacitidine for patients with primary and post-essential thrombocythemia/polycythemia vera myelofibrosis. Leukemia, 22, 965-70.

Rambaldi A, Dellacasa CM, Finazzi G, et al (2010). A pilot study of the Histone-Deacetylase inhibitor Givinostat in patients with JAK2V617F positive chronic myeloproliferative neoplasms. Br J Haematol, 150, 446-55.

Rampal R, Al-Shahrour F, Abdel-Wahab O, et al (2014). Integrated genomic analysis illustrates the central role of JAK-STAT pathway activation in myeloproliferative neoplasm pathogenesis. Blood, 123, 123-33.

Rampal R, Levine RL (2014). A primer on genomic and epigenomic alterations in the myeloproliferative neoplasms. Best Pract Res Clin Haematol, 27, 83-93.

Saeidi K (2016). Myeloproliferative neoplasms: Current molecular biology and genetics. Crit Rev Oncol Hematol, 98, 375-89.

Santos FP, Verstovsek S (2013). What is next beyond janus kinase 2 inhibitors for primary myelofibrosis?. Curr Opin Hematol, 20, 123-9.

Sasazawa Y, Sato N, Suzuki T, et al (2015). C-Mannosylation of thrombopoietin receptor $(\mathrm{c}-\mathrm{Mpl})$ regulates thrombopoietindependent JAK-STAT signaling. Biochem Biophys Res Commun, 468, 262-8.

Sauvageau M, Sauvageau G (2010). Polycomb group proteins: multi-faceted regulators of somatic stem cells and cancer. Cell Stem Cell, 7, 299-313.

Scherber R, Dueck AC, Johansson P, et al (2011). The myeloproliferative neoplasm symptom assessment form (MPN-SAF): international prospective validation and reliability trial in 402 patients. Blood, 118, 401-8.

Scheuermann JC, de Ayala Alonso AG, Oktaba K, et al (2010). Histone H2A deubiquitinase activity of the Polycomb repressive complex PR-DUB. Nature, 465, 243-7.

Shan Y, Gnanasambandan K, Ungureanu D, et al (2014). Molecular basis for pseudokinase-dependent autoinhibition of JAK2 tyrosine kinase. Nat Struct Mol Biol, 21, 579-84.

Singer J, Al-Fayoumi S, Ma H, et al (2014). Comprehensive kinase profile of pacritinib, a non-myelosuppressive JAK2 kinase inhibitor in phase 3 development in primary and post ET/PV myelofibrosis. Blood, 124, 1874.

Singh NR (2015). Genomic diversity in myeloproliferative neoplasms: focus on myelofibrosis. Transl Pediatr, 4, 107-15.

Sochacki AL, Fischer MA, Savona MR (2016). Therapeutic approaches in myelofibrosis and myelodysplastic/ myeloproliferative overlap syndromes. Onco Targets Ther, 9, 2273-86.

Stein BL, Cervantes F, Giles F, et al (2015). Novel therapies for myelofibrosis. Leuk Lymphoma, 56, 2768-78.

Tefferi A (2010). Novel mutations and their functional and clinical relevance in myeloproliferative neoplasms: JAK2, MPL, TET2, ASXL1, CBL, IDH and IKZF1. Leukemia, 24, 1128.

Tefferi A (2016). Myeloproliferative neoplasms: A decade of discoveries and treatment advances. Am J Hematol, 91, 50-8.

Tefferi A, Finke CM, Lasho TL, et al (2014a). U2AF1 mutations in primary myelofibrosis are strongly associated with anemia and thrombocytopenia despite clustering with JAK2V617F and normal karyotype. Leukemia, 28, 431-3.

Tefferi A, Guglielmelli P, Larson DR, et al (2014b). Long-term survival and blast transformation in molecularly annotated essential thrombocythemia, polycythemia vera, and myelofibrosis. Blood, 124, 2507-13; quiz 615.

Tefferi A, Lasho TL, Begna KH, et al (2015). A Pilot Study of the Telomerase Inhibitor Imetelstat for Myelofibrosis. N Engl J Med, 373, 908-19.

Tefferi A, Lasho TL, Finke CM, et al (2014c). CALR vs JAK2 vs MPL-mutated or triple-negative myelofibrosis: clinical, cytogenetic and molecular comparisons. Leukemia, 28, $1472-7$.

Tefferi A, Litzow MR, Pardanani A (2011). Long-term outcome of treatment with ruxolitinib in myelofibrosis. $N$ Engl J Med, 365, 1455-7.

Tefferi A, Pardanani A, Lim KH, et al (2009). TET2 mutations and their clinical correlates in polycythemia vera, essential thrombocythemia and myelofibrosis. Leukemia, 23, 905.

Vainchenker W, Constantinescu SN, Plo I (2016). Recent advances in understanding myelofibrosis and essential thrombocythemia. F1000Res, 5.

Vainchenker W, Kralovics R (2017). Genetic basis and molecular pathophysiology of classical myeloproliferative neoplasms. Blood, 129, 667-79.

Vannucchi AM, Bogani C, Bartalucci N, et al (2011). Inhibitors of PI3K/Akt and/or mTOR Inhibit the Growth of Cells of Myeloproliferative Neoplasms and Synergize with JAK2 Inhibitor and Interferon. Blood, 118, 3835-

Verstovsek S, Mesa RA, Gotlib J, et al (2012). A double-blind, placebo-controlled trial of ruxolitinib for myelofibrosis. N Engl J Med, 366, 799-807.

Yoshida K, Sanada M, Shiraishi Y, et al (2011). Frequent pathway mutations of splicing machinery in myelodysplasia. Nature, 478, 64-9.

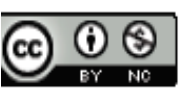

This work is licensed under a Creative Commons AttributionNon Commercial 4.0 International License. 